\title{
Erratum to: The p66Shc redox adaptor protein is induced by saturated fatty acids and mediates lipotoxicity-induced apoptosis in pancreatic beta cells
}

\author{
Annalisa Natalicchio ${ }^{1} \cdot$ Federica Tortosa $^{1} \cdot$ Rossella Labarbuta $^{1} \cdot$ Giuseppina Biondi $^{1}$. \\ Nicola Marrano ${ }^{1}$ - Emanuele Carchia ${ }^{2}$ - Anna Leonardini ${ }^{1}$ - Angelo Cignarelli ${ }^{1}$. \\ Marco Bugliani $^{3}$ - Piero Marchetti ${ }^{3}$ - Gian Paolo Fadini ${ }^{4}$ - Marco Giorgio ${ }^{5}$. \\ Angelo Avogaro ${ }^{4} \cdot$ Sebastio Perrini $^{1} \cdot$ Luigi Laviola $^{1} \cdot$ Francesco Giorgino $^{1}$
}

Published online: 1 September 2015

(C) Springer-Verlag Berlin Heidelberg 2015

\section{Erratum to: Diabetologia}

DOI 10.1007/s00125-015-3563-2

Unfortunately, the details of the high-fat diet given in the Methods section of this paper were incorrect. The correct details are given here.

At the age of 3 weeks, mice were randomised to a high-fat diet (HFD; purchased from Mucedola [Settimio Milanese, Milan, Italy] and consisting of $60 \%$ energy from hydrogenated palm fat) or continued on a standard diet for an additional 21 days.

The online version of the original article can be found at http://dx.doi.org/ 10.1007/s00125-015-3563-2.

Francesco Giorgino

francesco.giorgino@uniba.it

1 Department of Emergency and Organ Transplantation, Section of Internal Medicine, Endocrinology, Andrology and Metabolic Diseases, University of Bari Aldo Moro, Piazza Giulio Cesare, 11, 70124 Bari, Italy

2 IRGS Biogem, Ariano Irpino, AV, Italy

3 Department of Clinical and Experimental Medicine, Islet Cell Laboratory, University of Pisa, Pisa, Italy

4 Department of Medicine, Venetian Institute of Molecular Medicine, University of Padua, Padua, Italy

5 Department of Experimental Oncology, European Institute of Oncology, Milan, Italy 